\title{
Design of Multiband PIFA loaded with Split Ring Resonator
}

\author{
Parneet Kaur ${ }^{1}$, Garima Saini ${ }^{2}$ \\ ${ }^{1}$ (ECE, NITTTR, Punjab University, India) \\ 2 (Assistant Professor, NITTTR, Punjab University, India)
}

\begin{abstract}
In this paper, a multiband planar inverted-F antenna (PIFA) with high gain and appreciable bandwidth at the resonant frequencies is designed using metamaterial concept. The proposed PIFA without metamaterial is designed to resonate at $7.46 \mathrm{GHz}$ with return loss of $-23.71 \mathrm{~dB}$ and gain value $5.41 \mathrm{dBi}$. The designed antenna is having maximum possible bandwidth of $361.8 \mathrm{MHz}$. The proposed PIFA with split ring resonator (SRR) loading resonates at multiple frequencies i.e. $5.60 \mathrm{GHz}, 6.68 \mathrm{GHz}, 7.98 \mathrm{GHz}, 8.44 \mathrm{GHz}$ and $9.42 \mathrm{GHz}$. Return loss at $5.60 \mathrm{GHz}$ is $-22.48 \mathrm{~dB}$ and gain is $7.10 \mathrm{dBi}$. At $6.68 \mathrm{GHz}$ return loss is $-34.55 \mathrm{~dB}$ and gain is $7.68 \mathrm{dBi}$. Return loss is $-30.4 \mathrm{~dB}$ at $7.98 \mathrm{GHz}$ and gain is $4.21 \mathrm{dBi}$. At $8.44 \mathrm{GHz}$ return loss is $-15.23 \mathrm{~dB}$ and gain is $4.04 \mathrm{dBi}$. Return loss and gain values at $9.42 \mathrm{GHz}$ is $-16.88 \mathrm{~dB}$ and $4.77 \mathrm{dBi}$ respectively. After loading with metamaterial maximum bandwidth observed is of $736 \mathrm{MHz}$. Metamaterial loading has shown to increase the number of resonant frequencies of reference antenna with bandwidth and gain enhancement. It also had an impact on the antenna performance in terms of the return loss improvement and size reduction. Nicolson-Ross-Weir (NRW) method has been employed for verifying metamaterial characteristics of SRR. Design is simulated using HFSS Software.
\end{abstract}

Keywords: Multiband, Nicolson-Ross-Weir (NRW), PIFA, return loss, Split Ring Resonator (SRR).

\section{Introduction}

PIFA is widely used in mobile and portable applications because of its various merits such as low cost, low profile and attractive radiation patterns etc. However, it has limitation of narrow bandwidth and low gain. Over the years, several techniques have been proposed by researchers for bandwidth and gain enhancement of PIFA. The rapid development of electronics and wireless communications led to great demand for wireless devices that can operate at different standards such as universal mobile telecommunications system (UMTS), Bluetooth, wireless local-area network (WLAN) and also satellite communications[1].Therefore, it is required to design small size, high gain, and low cost antennas with appreciable bandwidth for multiband applications. There are a number of PIFA designs with different configurations to achieve single and multiple operations by using slots of different shapes. Truncated corner technique, meandered strips and meandered shapes have been used to create multiple band operations [2]. However, these techniques usually have a tradeoff with antenna bandwidth or gain while achieving multiband characteristics. Metamaterial loading has proved to be an advantageous approach for improving basic antenna features such as impedance matching, bandwidth, gain and efficiency along with miniaturization.

Metamaterials are the artificial materials having properties which may not be found in nature. These materials possess negative permeability $(\mu)$ and negative permittivity $(\varepsilon)$ that support the backward wave propagation of electromagnetic waves [3]. The invention of metamaterial started in the late 1960s. In 1967, Russian physicist Victor Georgievich Veselago studied the electrodynamics of substances with simultaneously negative values of dielectric permittivity $(\varepsilon)$ and magnetic permeability $(\mu)$ [3]. Later on J.B. Pendry and his colleagues, proved that the array of metallic wires can be used to obtain negative permittivity and split ring resonators for negative permeability [4]. Next a structure was fabricated, which was a composition of split ring resonator and thin wire and was named as LHM (left handed material) because the electric field (E), magnetic intensity $(\mathbf{H})$ and propagation vector $(\mathbf{k})$ were related by a left-hand rule. Apart from exhibiting negative refractive index, metamaterials also presents some interesting properties such as the reversal of the Snell Law, Doppler Effects, and Cherenkov radiation etc.

A compact SRR loaded antenna was presented in [5]. It was seen that the gap between patch and SRR plays an important role in determining the resonant frequency of antenna. In [6], gain of the antenna was improved by placing metamaterial slab over the patch. Power radiated by antenna can be improved by employing DNG material [7]. In [8], miniaturized annular patch resonator was designed by partially loading it with metamaterial. In [2], slots are made on the patch to obtain multiband PIFA for WLAN and WiMAX application. But the gain achieved from the structure could reach upto maximum of 5dbi. In [9] multiband patch antenna filled with metamaterial structure over a metamaterial substrate is investigated and designed. Designed antenna however has lower gain and efficiency compared to a patch antenna with a metamaterial substrate or patch antenna filled with metamaterial structures and placed over FR4 substrate. A printed monopole antenna is proposed [10], in which broadband dual mode feature is obtained using metamaterial loading. Split Ring 
Resonator (SRR) is a novel design consisting of two concentric metallic rings with a split on opposite sides. SRR can result in an effective negative permeability over a particular frequency region. They exhibit magnetic resonance at certain frequencies and hence are called as resonator. Basically, SRR is a $L C$ tank circuit having equivalent inductance " $L$ " and the capacitance " $C$ " between two concentric rings resonating at particular frequency [11]. SRRs are well known in metamaterials since they can provide negative permeability that can create a stopband response at the resonant frequency and also produce new bands of operation [12].

In this work the multiband PIFA with high gain, reduced size and wider bandwidth is proposed using split ring resonator (SRR). SRR is printed on the substrate of reference antenna. The characteristics of PIFA are studied without SRR and with SRR loading. This paper is structured into five sections. The detailed geometrical structure and design of the proposed PIFA without SRR and with SRR loading is presented in Section 2 and 3 respectively. In Section 4, the metamaterial properties of SRR are verified using effective medium theory. The simulation results of unloaded and loaded PIFA are also presented in this section. Finally, the paper is concluded in Section 5.

\section{Proposed PIFA Antenna} equation (1).

The design started with the conventional patch length and width which can be calculated by using

$$
L+W=\frac{\lambda}{4}
$$

Where $\lambda$ is the wave length, $L$ and $W$ are the length and width of the top patch. The final dimensions of antenna are calculated to be $74 \times 60 \times 7 \mathrm{~mm} 3$, to make it resonate at $7.46 \mathrm{GHz}$. The patch is having dimensions of $44 \times 50 \mathrm{~mm} 2$ and shorting plate is having a width of $5 \mathrm{~mm}$. Fig1.depicts the geometrical structure of proposed PIFA without metamaterial loading. The dielectric substrate used for design is FR-4, with dielectric constant 4.4 and thickness $1.5748 \mathrm{~mm}$. The antenna is fed using $50 \Omega$ microstrip transmission line of width $5 \mathrm{~mm}$.

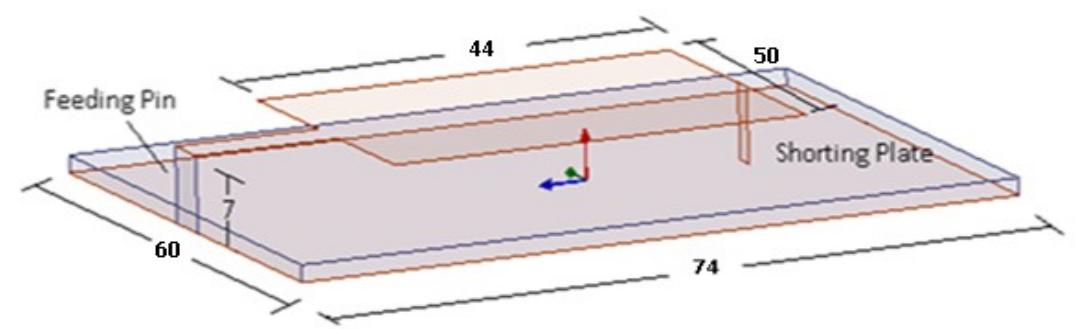

Fig. 1: The 3D view and detailed dimensions of the proposed antenna without metamaterial

\section{Proposed PIFA with SRR}

Proposed structure consists of a short circuited patch at a distance of $3 \mathrm{~mm}$ from ground and circular SRR's printed over the substrate. SRR structure is used to exhibit negative permeability. Fig. 2 shows the geometrical structure of SRR unit cell. The radius of outer ring $(\mathrm{r} 2)=4 \mathrm{~mm}$ and the radius of inner ring $(\mathrm{r} 1)=2$ $\mathrm{mm}$. The separation between inner and outer ring $(\mathrm{d})=1 \mathrm{~mm}$, gap in the ring $(\mathrm{g})=1 \mathrm{~mm}$ and width of the circular ring $(c)=1 \mathrm{~mm}$. Fig. 3 shows the detailed dimensions of PIFA with SRR loading. The dimensions of the PIFA with SRR structure is 65 X 46 X $3 \mathrm{~mm} 3$. Patch is having dimensions of 40 X $32 \mathrm{~mm} 2$. Shorting and feeding pins are having the same dimensions as reference antenna. FR-4 substrate with the thickness as 1.5748 $\mathrm{mm}$ and dielectric constant value 4.4 is used in the design.

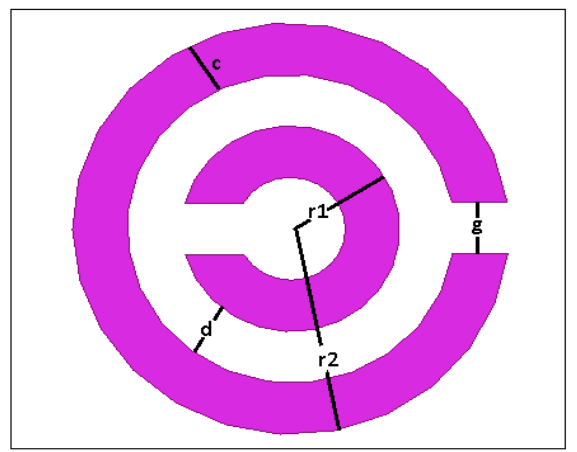

Fig. 2 Geometrical structure of metamaterial SSR unit cell. 


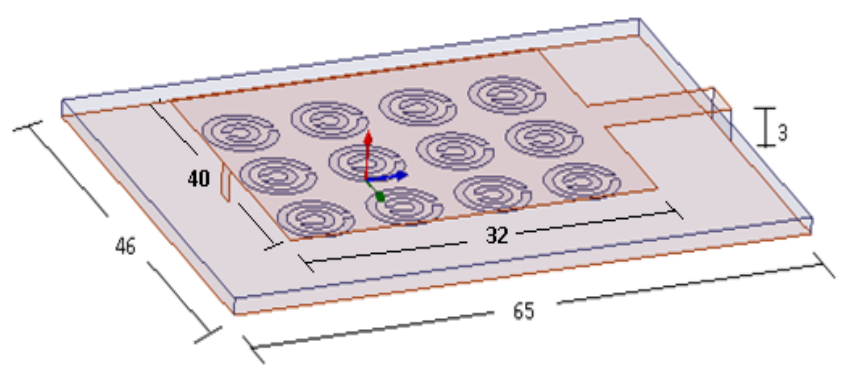

Fig. 3: The 3D view and detailed dimensions of the proposed antenna with SRR

\section{Simulation results and metamaterial verification}

In this section, the metamaterial properties of SRR are verified using effective medium theory. Nicolson-Ross-Weir method (NRW) has been employed for verifying that SRR metamaterial structure possesses negative values of Permeability within the operating frequency ranges. Fig. 4 shows the reflection coefficient $\left(S_{11}\right)$ and transmission coefficient $\left(S_{21}\right)$ characteristics of the SRR. It shows that the SRR resonate at $10.9 \mathrm{GHz}$.

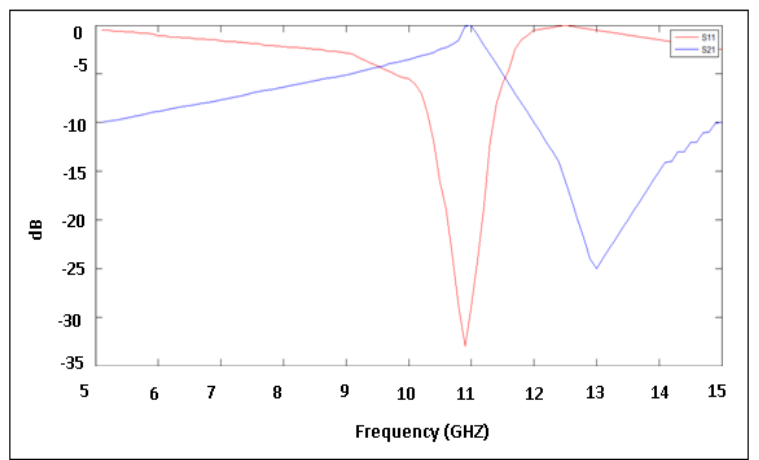

Fig. 4: S-parameters $\left(S_{11}\right)$ and $\left(S_{21}\right)$ of the SRR structure

The effective medium parameters are derived from the reflection and transmission coefficient parameters (S-parameters) using Nicolson-Ross-Weir (NRW) approach. The expressions of Equations (2) and (3) are used for calculating these values [13].

$$
\begin{aligned}
& \mu_{r}=\frac{2}{j k_{0} d} \frac{1-V_{2}}{1+V_{2}} \\
& \varepsilon_{r}=\frac{2}{j k_{0} d} \frac{1-V_{1}}{1+V_{1}}
\end{aligned}
$$

where $k_{0}$ is wave number, $d$ is the thickness of substrate, $V 1$ and $V 2$ are the composite terms to represent the addition and subtraction of S-parameters. The values of $V 1$ and $V 2$ are estimated using Equations (4) and (5) [13].

$$
\begin{aligned}
& V_{1}=S_{21}+S_{11} \\
& V_{2}=S_{21}-S_{11}
\end{aligned}
$$

Fig. 5 indicates the permeability characteristics $(\mu)$ of SRR unit cell. This structure exhibits real negative permeability $\left(\mu_{\mathrm{r}}\right)$ which indicates single negative that is mu negative (MNG) characteristics of SRR structure. By using the obtained S-parameters, above mathematical equations and MATLAB code the metamaterial characteristics of SRR are verified. 


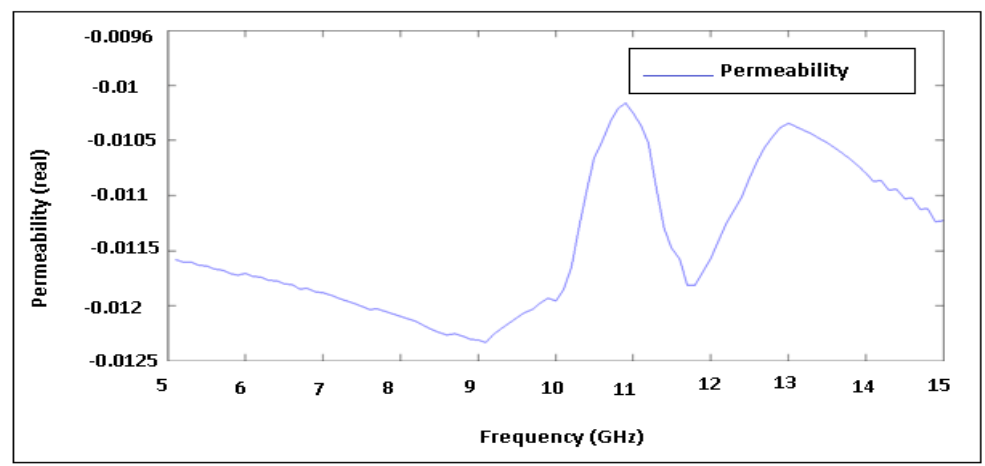

Fig.5: Permeability $\left(\mu_{\mathrm{r}}\right)$ characteristics of SRR.

The return loss $(S 11)$ characteristic of an unloaded PIFA is shown in Fig. 6. PIFA without loading resonates at $f_{\mathrm{r}}=7.46 \mathrm{GHz}$ with return loss of $-23.71 \mathrm{~dB}$. The bandwidth achieved for this structure is $361.8 \mathrm{MHz}$. Fig.7 depicts the gain plot for reference antenna which is having a value of $5.41 \mathrm{dBi}$

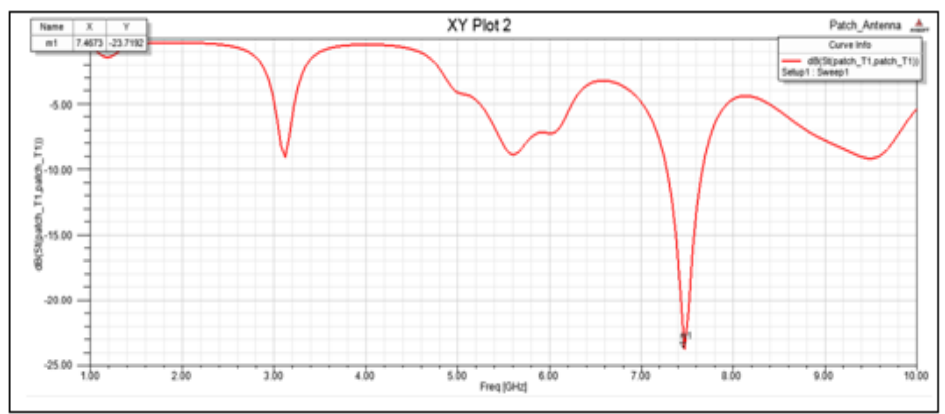

Fig. 6: Simulated return loss results for proposed PIFA without metamaterial

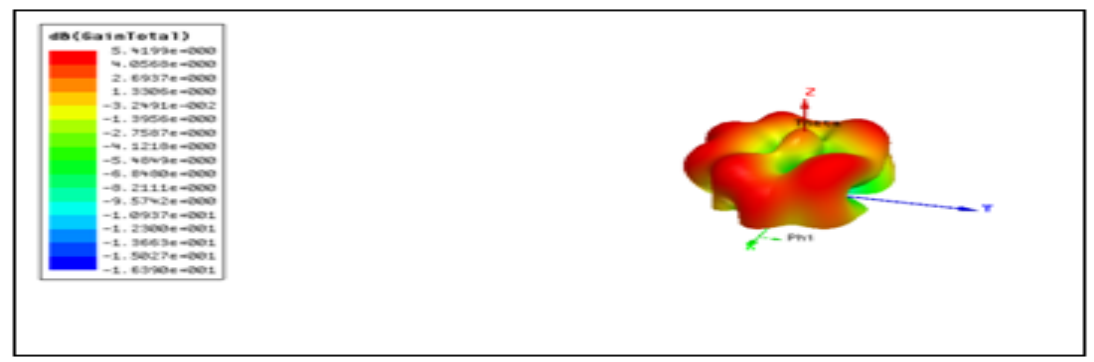

Fig. 7: Total gain of reference antenna

Fig. 8 shows simulated return loss versus frequency results for PIFA with SRR loading. It is seen that the proposed antenna structure resonates at multiple frequencies. Also after loading, the antenna shows reduction in its resonant frequency and thus miniaturization is accomplished in proposed structure. Fig. 9 shows the gain values at resonant frequencies. Maximum gain value achieved for the structure is $7.68 \mathrm{dBi}$ at $6.68 \mathrm{GHz}$. The proposed antenna shows $32.65 \%$ reduction in the size compared to the reference antenna. Table I shows the values of antenna parameters such as return loss, gain and bandwidth values at different resonant frequencies.

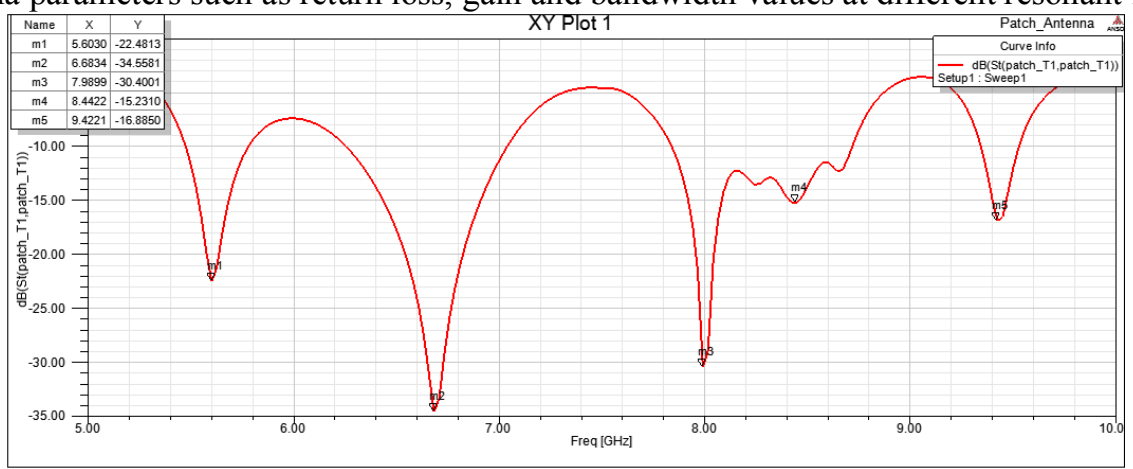

Fig. 8: Simulated return loss results for proposed PIFA with SRR 


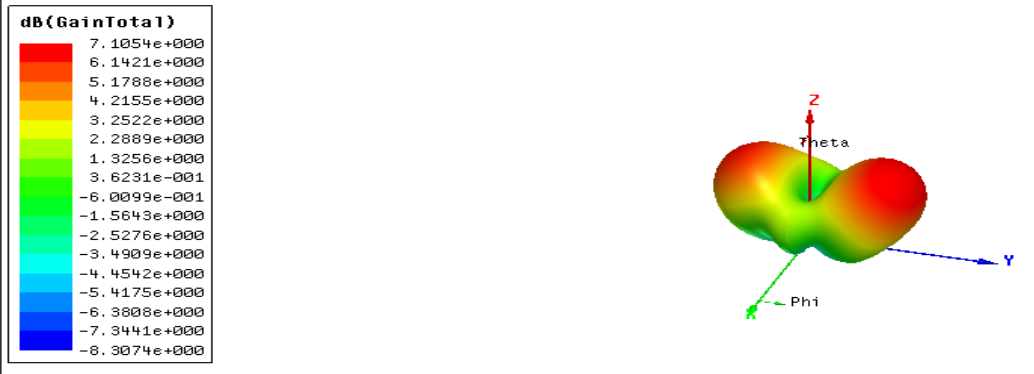

(a)

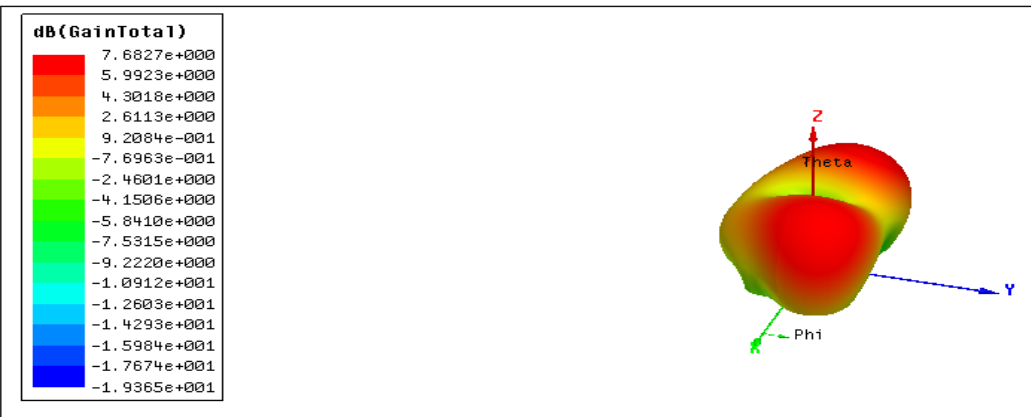

(b)
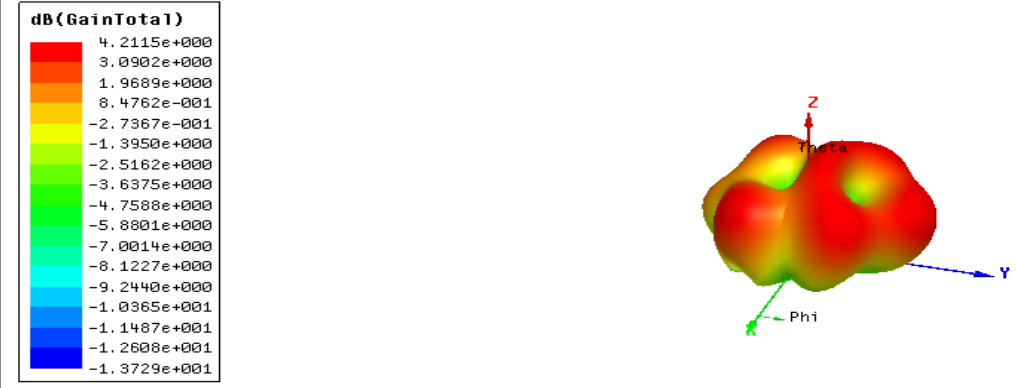

(c)
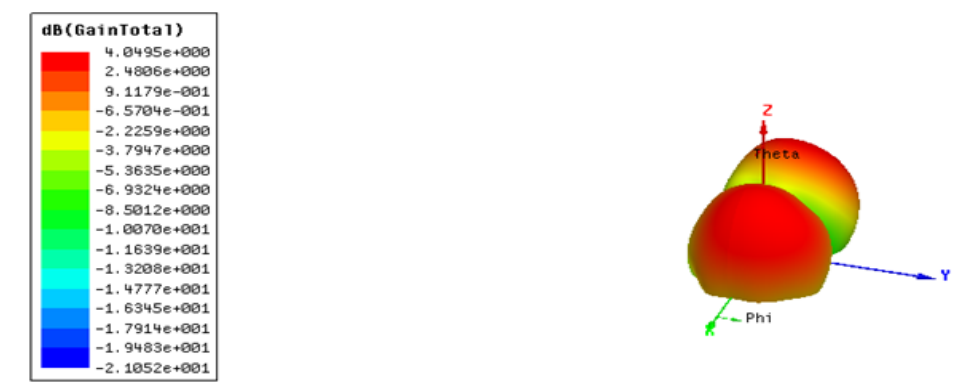

(d)
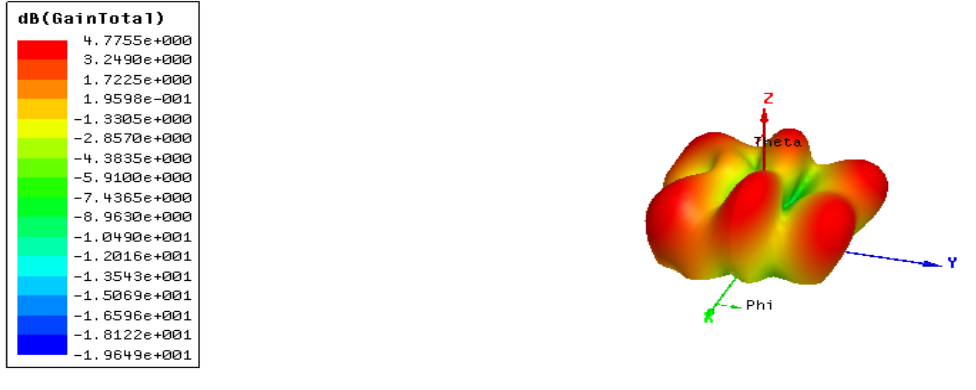

(e)

Fig.9: Total gain of PIFA with metamaterial (a) at $5.60 \mathrm{Ghz}$ (b) $6.68 \mathrm{Ghz}$ (c) $7.98 \mathrm{Ghz}$ (d) $8.44 \mathrm{Ghz}$ (e) 9.42 Ghz 
TABLE I: Antenna parameters with SRR loading

\begin{tabular}{|l|l|l|l|l|}
\hline BAND & FREQUENCY IN (GHz) & $\begin{array}{l}\text { MIN. RETURN LOSS } \\
(\mathrm{S} 11) \text { IN dB }\end{array}$ & GAIN IN (dBi) & $\begin{array}{l}\text { BANDWIDTH IN } \\
(\mathrm{MHz})\end{array}$ \\
\hline I & 5.60 & -22.48 & 7.10 & 251 \\
\hline II & 6.68 & -34.55 & 7.68 & 678 \\
\hline III & 7.98 & -30.40 & 4.21 & 736 \\
\hline IV & 8.44 & -15.23 & 4.04 & 736 \\
\hline V & 9.42 & -16.88 & 4.77 & 201 \\
\hline
\end{tabular}

\section{Conclusion}

From the simulated results it is found that the parameters of the proposed antenna with SRR loading increases significantly in comparison to PIFA alone. The main advantages are basically related to the multiband behavior achieved, high gain and wider bandwidth. The resonant frequency of proposed antenna is also reduced to lower value after loading with SRR and hence provided miniaturization effect. The proposed structure finds its usability in mobile application and satellite communication.

\section{References}

[1] Rowell, C. R., Murch and R. D., A capacitively loaded PIFA for compact mobile telephone handsets, IEEE Transaction on Antennas and Propagation, 45(5), 1997, 837-842.

[2] Hattan F. AbuTarboush, R. Nilavalan, Thomas Peter, and S. W. Cheung, Multiband Inverted-F Antenna with Independent Bands for Small and Slim Cellular Mobile Handsets, IEEE Transaction on Antennas and Propagation, 59(7), 2011, $2636-2645$.

[3] V. G. Veselago, The Electrodynamics of Substances with Simultaneously Negative Values of $\varepsilon$ and $\mu$, Soviet Physics Uspekhi, 10(4), 1968, 509-514.

[4] Bimal Garg, Rahul Dev Verma and Ankit Samadhiya, Design of Rectangular Microstrip Patch Antenna Incorporated with Innovative Metamaterial Structure for Dual band operation and Amelioration in Patch Antenna Parameters with Negative $\mu$ and $\varepsilon$, International Journal of engineering and technology,1(3),2012, 205-216.

[5] R. Pandeeswari, Singaravelu Raghavan, and Keloth Ramesh, A Compact Split Ring Resonator Loaded Antenna, Progress In Electromagnetics Research Symposium Proceedings, Moscow, Russia,2012,37-40.

[6] Attia, H., L. Yousefi, M. M. Bait-Suwailam, M. S. Boybay, and O. M. Ramahi, Enhanced gain microstrip antenna using engineered magnetic superstrates,IEEE Antennas and Wireless Propagation Letters, 8,2009,1198-1201.

[7] Joshi, J. G., S. S. Patnaik, S. Devi, M. R. Lohokare, and C. Vidyasagar, Offset fed diamond shaped split ring (DSRR) planar metamaterial antenna, Applied electromagnetics conference, 2009, 1-4.

[8] Mahmoud and S. F., A new miniaturized annular ring patch resonator partially loaded by a metamaterial ring with negative permeability and permittivity, IEEE Antennas and Wireless Propagation Letters, 3(1), 2004,19-22.

[9] M.S. Soudi and H.M. Elkamchouchi, A proposed multiband metamaterial antenna based on a circular air slotted sievenpipper mushroom unit cell, IEEE, Middle East Conference on Antennas and Propagation (MECAP),Cairo,Egypt,2010,1-7. [10] M.A. Antoniades and G.V. Eleftheriades, A broadband dual-mode monopole antenna using NRI-TL metamaterial loading, IEEE Antennas and Wireless Propagation Letters, 8, 2009, 258-261.

[11] J. B. Pendry, A. J. Holden, D. J. Robbins and W. J. Stew-art, Magnetism from Conductors and Enhanced Nonlinear Phenomena, IEEE Transactions on Microwave Theory and Techniques, 47(11), 1999, 2075- 2084.

[12] Elena Pucci, Eva Rajo-Iglesias, Malcolm Ng Mou Kehn and Oscar Quevedo-Teruel, Enhancing the Efficiency of Compact Patch Antennas Composed of Split-Ring Resonators by Using Lumped Capacitors, IEEE Antennas and Wireless Propagation Letters, 11, 2012, 1362-1365.

[13] Jayant G. Joshi, Shyam S. Pattnaik, Swapna Devi and Mohan R. Lohokare, Bandwidth Enhancement and Size Reduction of Microstrip Patch Antenna by Magnetoinductive Waveguide Loading, Wireless Engineering and Technology, 2(2), 2011, 37-44. 